\title{
Sex differences in patients with mesial temporal lobe epilepsy
}

\author{
Ivanka Savic, Jerome Engel Jr
}

Department of Human

Brain Research, Karolinska Institute

I Savic

Department of Neurology, Huddinge University Hospital, Stockholm, Sweden

I Savic

Department of

Neurology

$\mathrm{J}$ Engel

Department of Neurobiology

J Engel

Brain Research Institute, UCLA School of Medicine, Los Angeles, California, USA

$\mathrm{J}$ Engel

Correspondence to: Dr Ivanka Savic, Karolinska Institute, Department of Neurology, Huddinge University Hospital, 14186 Huddinge, Sweden.

Telephone 00468585800 00 ; fax 004687744822 ; email

ivanka.savic-berglund@ neuro.ki.se

Received 22 January 1998 and in final form

7 July 1998

Accepted 23 July 1998

\begin{abstract}
Possible sex differences in the pattern of interictal hypometabolism were investigated, and also seizure spread in patients with mesial temporal lobe epilepsy $(n=48)$ and hippocampal sclerosis (MTLE). Male patients $(n=21)$ more often had a frontal lobe hypometabolism ipsilateral to the seizure onset $(p<0.0001)$ and a spread of epileptiform activity to this region $(p=0.001)$. By contrast, female patients more often exhibited hypometabolism $(p=0.0052)$ and an ictal spread to the contralateral temporal lobe $(p=0.0097)$. These findings suggest sex differences in spatial distribution of brain dysfunction in MTLE, perhaps reflecting sexual dimorphism in regional cerebral connectivity. (F Neurol Neurosurg Psychiatry 1998;65:910-912)
\end{abstract}

Keywords: sex; brain; epilepsy; glucose metabolism; positron emission tomography

Sex differences are suggested in the functional and anatomical organisation of the human brain. ${ }^{1-9}$ Males perform better in visuospatial tasks, which can be attributed to their higher relative grey matter volume in the right temporoparietal area $^{2}$ and, perhaps, stronger connections with the ipsilateral frontal cortex, which is coactivated during visuospatial tasks. ${ }^{7}$ Females show better verbal abilities and have a larger relative grey matter volume in the planum temporale. ${ }^{6}$ They also activate both hemispheres during language tasks, and show a better recovery after lesions in the language mediating structures. ${ }^{89}$

These findings have led to the hypothesis about sex differences in interhemispheric and intrahemispheric cerebral connections, recently supported by a finding of a higher number of interhemispheric correlations in regional cerebral metabolite rate for glucose (rCMRglu) in women and intrahemispheric correlations in men. ${ }^{3}$

Mesial temporal lobe epilepsy associated with hippocampal sclerosis (MTLE) is pathophysiologically a well defined condition, often associated with complex partial seizures of mesial temporal lobe origin. ${ }^{10}$ Despite the stereotyped pathology, patients with MTLE show a variable seizure semiology, probably due to differences in seizure spread. ${ }^{11}$
About $80 \%$ of patients with MTLE show an interictal hypometabolism within the areas of seizure onset. ${ }^{10}$ As seizure semiology and spread, its regional pattern varies in these patients.

Because seizure spread as well as the anatomical pattern of epileptogenic brain areas may result from inherently preferential intracerebral connections, and because these connections may differ between male and female subjects, we postulated the existence of sex differences in the pattern of hypometabolism in patients with MTLE.

The distribution of glucose hypometabolism was retrospectively analysed in 27 female and 21 male patients with postsurgically defined MTLE. The results were related to the data on regional spread patterns of ictal epileptiform activity recorded with EEG telemetry.

\section{Methods}

PATIENTS

Among patients with mesial temporal lobe seizures who underwent surgical treatment at the UCLA Seizure Disorder Center between 1993 and 1997 we included patients with: (1) hippocampal sclerosis and absence of other brain pathology on morphological analysis of the resected tissue; (2) clinically and electrophysiologically well defined and stereotyped onsets and evolution of seizures. Exclusion criteria were: (1) diffuse or regional cerebral abnormality outside the area of seizure onset, determined by neuropsychological tests, EEG recordings, or MRI (1.5 tesla GE scanner, 3D SPGR sequences, TR $40 \mathrm{~ms}$, TE $9 \mathrm{~ms}$, Flip angle 9, slice thickness 1.7-1.9 mm); (2) secondarily generalised seizures (>three/year, or within 2 weeks before PET); (3) bilateral abnormality on the Wada test. Inclusion criteria were met by 48 patients ( 21 males). The area of seizure onset was right sided in 11 subjects in each group. Apart from having seizures the patients were healthy.

POSITRON EMISSION TOMOGRAPHY (PET) In all the patients PET was preceded by a habitual seizure. Measurements with $\left[{ }^{18} \mathrm{~F}\right]-$ FDG PET were conducted according to the previously described procedure. ${ }^{13-15}$ Anatomical regions of interest (ROIs), with minimum size of $1.5 \mathrm{~cm}^{3}$, were drawn using a standardised template of anatomical regions. Three 
Results from (A) PET and (B) EEG analyses. Some patients had several hypometabolic areas outside the seizure onset region

\begin{tabular}{|c|c|c|c|c|c|c|}
\hline $\operatorname{Sex}$ & Il frontal & Cl frontal & Cl temporal & Il parietal & Cl parietal & No spread \\
\hline \multicolumn{7}{|c|}{ (A) Regions with hypometabolism outside the epileptogenic region: } \\
\hline Male $(n=21)$ & $13+$ & 4 & $3+t$ & 2 & 1 & 8 \\
\hline Female $(n=27)$ & 6 & 2 & 14 & 4 & 3 & 10 \\
\hline \multicolumn{7}{|c|}{ (B) Spread of epileptiform activity during seizures: } \\
\hline Male $(\mathrm{n}=21)$ & $13^{\star}$ & 3 & $5^{\star \star}$ & 2 & 2 & 4 \\
\hline Female $(n=27)$ & 4 & 1 & 17 & 6 & 3 & 7 \\
\hline
\end{tabular}

Il=ipsilateral; $\mathrm{Cl}=$ contralateral. ${ }^{\star} \mathrm{p}=0.001 ;{ }^{\star \star} \mathrm{p}=0.0097 .+\mathrm{p}<0.0001 ; \mathrm{\dagger} \mathrm{p}=0.0052$.

separate ROIs were delineated in the temporal lobes (anterior, mesial, and lateral), and seven in the frontal lobes (the orbitofrontal, insular, dorsolateral frontal, and frontopolar cortex, the anterior cingulate, and the precentral and postcentral gyrus). ${ }^{13}$ The ROIs were collapsed into several main gross areas-lobes (the frontal, occipital, and parietal lobe ipsilateral to the seizure onset, the contralateral frontal, temporal, and occipital cortex.

The average local count rate from each ROI was divided by the mean value of the whole suprasylvian brain, including the ventricles, yielding ROI:brain ratios. ${ }^{15}$ Regions of interest with ratios outside the $95 \%$ confidence interval of 14 sex matched controls (seven males) were then identified, enabling selection of gross areas with hypometabolic ROIs. The regional metabolic ratios of controls showed no sex differences, which allowed the use of mean values. During evaluation of PET data the investigator was unaware of the sex, and definite location of the epileptogenic region.

\section{EEG RECORDINGS}

The EEG recordings were evaluated for clinical purposes, before the study was conducted, by consensus of three electrophysiologists. Withdrawal of antiepileptic medication during EEG telemetry monitoring did not differ between male and female patients. The location of ictal discharge was evaluated sequentially during 15 second epochs. The spread of ictal discharge was defined as any significant change in the frequency and composition of the ongoing EEG that was sustained, and underwent evolution. Two aspects were taken into consideration: (1) the location of the first sign of spread; (2) the regions involved in the spread during the entire seizure. The localisation of spread was evaluated using the same classification of gross areas as in the analyses of PET data. The sex comparisons considered all the gross regions exhibiting epileptiform activity during the course of seizures.

\section{STATISTICS}

Possible sex differences in rCMRglu and pattern of seizure spread were evaluated by $\chi^{2}$ test. Sex differences in the mean hypometabolism within and outside the epileptogenic temporal lobe were tested with unpaired Student's $t$ tests. Finally, the demographic data (age, age at seizure onset, duration of seizures, medication) were compared using Mann-Whitney statistics. The level of significance was taken as $\mathrm{p}<0.05$ after the Bonferroni correction for the multiple comparisons.

\section{Results}

Male patients had a significantly higher incidence of frontal lobe hypometabolism ipsilateral to the area of seizure onset than female patients $(p<0.0001$, table $)$. They also more often showed spread of epileptiform activity to the ipsilateral frontal lobe $(\mathrm{p}=0.001)$. Notably, no sex difference was found in the frontal lobe contralateral to the area of seizure onset, which presumably was not involved in the epileptogenic process (the ROI:cortex ratio was 1.2 (SD 0.02) in both groups). Female patients more often exhibited a reduced metabolic ratio in the contralateral temporal lobe $(p=0.0052)$ and a spread of epileptiform activity to this area $(p=0.0097)$. Hypometabolism outside the epileptogenic zone in male patients was most often found in the ipsilateral orbitofrontal $(n=5)$ and frontopolar cortex $(n=5)$, and in females in the contralateral mesial temporal structures $(n=7)$.

The metabolic ratio in the area of seizure onset was 0.805 (SD 0.07) in male and 0.808 (SD 0.08) in female patients; the corresponding ratios of hypometabolic ROIs outside the area of onset were 0.870 (SD 0.03) and 0.867 (SD 0.03). The mean age (33 (SD 7) v 30 (SD 7 ), age at seizure onset (16 (SD 10) $v 10$ (SD 11), duration of seizures (16 (SD 12) $v 20$ (SD 12)), and the antiepileptic medication were similar in the two groups of patients, as was the number of patients with hypometabolism within $(n=41)$ and outside $(n=30)$ the epileptogenic zone.

In accordance with previous data, $80 \%$ of patients are reported seizure free 1-3 years after surgery. ${ }^{10}$

\section{Discussion}

METHODOLOGICAL CONSIDERATIONS

Because PET and MRI were not coregistered, possible partial volume effects were not corrected. Each ROI was delineated on several slices. Also, patients with MR abnormalities outside the epileptogenic zone were excluded, as were patients with visible hippocampal changes in the contralateral temporal lobe. Two male patients had slighly smaller contralateral hippocampi than normal. Because volumetric mesurements were not available, possible sex differences in hippocampal atrophy cannot be excluded, although the Wada tests and neuropsychological battery indicated unilateral abnormalities, and bilateral interictal EEG spikes were found only in two male patients. A finding of bilateral hippocampal sclerosis in females would, however, be in favour of the present findings. 
The scalp-sphenoidal EEG is not the optimal method for tracing seizure spread. Possible effects of its low resolution were minimised by using large regions in the comparisons, and by including all the regions involved in spread of the seizure. It is unlikely that low resolution would result in a systematically different spread pattern in males $v$ females.

IMPLICATIONS OF THE GENERATED DATA

Both the interictal hypometabolism and the pattern of seizure spread is thought to reflect epilepsy related synaptic reorganisation. ${ }^{13}{ }^{14}$ Differential findings may be due to inherent sexual dimorphism in cerebral connectivity. This interpretation is suggested by the intrahemispheric seizure spread and location of hypometabolism in male and corresponding interhemispheric dominance in female patients. The results are thus not surprising when considering available data on sexual dimorhism in healthy subjects. The present findings therefore encourage future studies on possible sex differences in partial epilepsy. Such studies may be of general interest, as partial epilepsy to some extent can be regarded as a human model for studies on cerebral connectivity.

This study was supported by the Swedish Medical Research Gren Center Foundations, The Swedish Institute, The Swedish Medical Society, and NIH grants NS-02808, NS-15654, NS-33310, and GM-24839. We acknowledge Sandra Dewer and Jody Schmidt for technical assistance.
1 McGlone J. Sex differences in human brain asymmetry: a critical survey. Behavioural Brain Science 1980;3:215-63.

2 Schaepfer TE, Harris GJ, Tien AY, et al. Structural differences in the cerebral cortex of healthy female and male subjects: a magnetic resonance imaging study. Psychiatry Res 1995;61:129-35.

3 Azari NP, Rapoport CL, Grady CL, et al. Gender differences in correlations of cerebral glucose metabolic rates in young normal adults. Brain Res 1992;574:198-208.

4 Jancke L, Staiger JF, Schlaug G, et al. The relationship Jancke L, Staiger JF, Schlaug G, et al. The relationship between corpus callosum s.

5 Shaywitz SA, Shaywitz Se, Pugh KR, et al. Sex differences in the functional organization of the brain for language. Nature 1995;373:607-9.

6 Harasty J, Double KL,Halliday GM, et al. Languageassociated cortical regions are proportionally larger in the female brain. Arch Neurol 1997;54:171-6.

7 Nobre AC, Sebestyen GN, Giterman DR, et al. Functional localization of the system for visuospatial attention using positron emission tomography. Brain 1997;120:515-33.

8 Trenerry MR, Jack CR Jr, Cascino GD, et al. Gender diferences in post temporal lobectomy verbal memory and relationships between MRI hippocampal volumes and preoperative verbal memory. Epilepsy Res 1995;20:69-75.

9 McGlone J. Sex differences in the cerebral organization of verbal functions in patients with unilateral lesions. Brain 1977;100:775-93.

10 Engel Jr J. Seizures and epilepsy. Philadelphia: F A Davis, $1989 ; 190-210$.

11 Wieser HG, Williamson PD. Ictal semiology. In: Engel J Jr. Surgical treatment of the epilepsies. 2nd ed. New York: Raven Press, 1003;161-71.

12 Savic I, Altshuler L, Baxter L, et al. Pattern of interictal hypometabolism in $\left[{ }^{18} \mathrm{~F}\right] \mathrm{FDG}$ PET reflects prior seizure semiology. Arch Neurol 1997;54:129-36.

13 Savic I, Altshuler L, Passaro E, et al. Impaired cerebellar glucose metabolism in partial epilepsy. Epilepsia 1996;37: glucose

14 Mazziotta JC, Phelps ME, Plummer D. Optimization and standardization of anatomical data in neurobehavioural investigations using positron CT. F Cereb Blood Flow Metab 1983;3:266-7

15 Theodore W, Fishbein D, Dubinsky R. Patterns of cerebral glucose metabolism in patients with partial seizures. Neurology 1988;38:1201-6. 